\title{
Apoio à Elaboração de Propostas de Projetos de Software através de um Ambiente de Gestão do Conhecimento
}

\author{
Viviane Costa, Ana Regina Rocha \\ COPPE / UFRJ - Programa de Engenharia de Sistemas e Computação \\ Caixa Postal 68511 - CEP 21945-970 - Rio de Janeiro - RJ \\ Tel: 21 2562-8675 / Fax: 21 2562-8676 \\ vivic@attglobal.net; darocha@cos.ufrj.br
}

\begin{abstract}
Resumo
Esse artigo discute uma abordagem sobre como a gestão do conhecimento pode apoiar a melhoria do Processo de Fornecimento de Software definido na ISO/IEC 12207 (1998) e na NBR ISO 10006 (2000). TecProposal é uma ferramenta de apoio ao aprendizado organizacional sobre as atividades, Iniciação e Preparação da Resposta, do Processo de Fornecimento de Software. A ferramenta foi desenvolvida em plataforma de internet e integrada ao ambiente TecKnowledge, configurado a partir do meta-ambiente CORE-KM.
\end{abstract}

Palavras-Chave: Processo de Fornecimento de Software, Gestão do Conhecimento, Aprendizado Organizacional e Qualidade do Processo

\section{Abstract}

This paper discusses how knowledge management can support the software systems supply process improvement, as defined in ISO/IEC 12207 (1998) and NBR ISO 10006 (2000). TecProposal is a tool that supports organizational learning in Initiation and Response to a Request for Proposal from the software systems supply process. The tool is web-based and it was integrated to the knowledge management environment, TecKnowledge, a CORE-KM configuration developed for a Software House.

Keywords: Software Systems Supply Process, Knowledge Management, Learning Organization e Software Quality Process

\section{Introdução}

Propostas de projetos de software são o marco inicial da negociação entre o cliente e o fornecedor desses serviços. Formalizam o entendimento do fornecedor de software sobre os requisitos inicialmente levantados pelo adquirente, levando em consideração políticas e outros regulamentos das organizações envolvidas na negociação. Essa formalização procura garantir o entendimento mútuo sobre o que foi pedido e o que foi sugerido como solução para o problema levantado pelo cliente. Na proposta, geralmente, são descritos: a definição do problema, estimativas de esforço, de cronograma e de custos, responsabilidades, estratégia de gerência de projeto etc. Portanto, pode-se considerar as propostas de projetos como guias pelos quais o negócio desenvolvimento de software é conduzido [11].

Organizações vivem crescente competitividade de mercados e procuram maximizar as chances de acordo com o cliente ao negociarem projetos. Desse modo, essas organizações precisam reutilizar e melhorar o conhecimento que possuem em elaborar propostas, objetivando o melhor entendimento do cliente sobre o que está sendo proposto.

Iniciativas que apóiam melhorias no processo de desenvolvimento de software, como o modelo CMM e as Normas ISO 9000 e ISO/IEC 15504 (SPICE), oferecem, implicitamente, a criação de estruturas de gestão do conhecimento. Organizações que se baseiam em qualquer uma dessas iniciativas podem gerenciar e distribuir conhecimento sobre o desenvolvimento de software, visando reduzir seus custos e prover a eficiência da equipe envolvida. A norma 
NBR ISO 10006 (2000) recomenda que sejam utilizados, em todas as atividades do processo, a experiência e os dados históricos provenientes de projetos anteriores.

Desta forma, esse trabalho sugere uma abordagem que se baseia na reutilização do conhecimento e de experiências anteriores para possibilitar a melhoria da qualidade de processos organizacionais.

O grupo de Engenharia de Software da COPPE/UFRJ está desenvolvendo uma infraestrutura para apoiar a gestão do conhecimento em processos organizacionais. A aplicação proposta nesse artigo, TecProposal, é parte dessa infra-estrutura maior e tem como objetivo apoiar utilização do conhecimento nas atividades Iniciação e Preparação da Resposta do Processo de Fornecimento de Software definido na ISO/IEC 12207 (1998) e na NBR ISO $10006(2000)$.

\section{Gestão do Conhecimento}

Conhecimento tem sido considerado o patrimônio que proporciona vantagem competitiva no mercado a uma organização e a mantém nessa condição. Esse conhecimento representa o capital intelectual de uma empresa e está distribuído entre os diversos indivíduos que a compõem. Sendo assim, para uma organização, perder um profissional pode significar a perda de conhecimento e de dinheiro. A fim de garantir o conhecimento estratégico, as empresas vêm investindo em processos de geração, armazenamento e transferência de conhecimento [21].

A gestão do conhecimento tem como objetivo a captura e a representação do conhecimento organizacional, visando facilitar o acesso, o compartilhamento e a reutilização do conhecimento [7]. A gestão do conhecimento é composta por todo o processo de descobrimento, aquisição, criação, disseminação e utilização do conhecimento, permitindo a criação da organização do aprendizado [2].

Sistemas de gestão do conhecimento proporcionam apoio automático a específicas fases do processo de gestão do conhecimento, a partir dos objetivos organizacionais. Além disso, ao definir esses sistemas, a organização deve considerar os aspectos humanos, sociais e técnicos, integrando os processos organizacionais ao de gestão do conhecimento. O sucesso de um sistema de gestão do conhecimento depende da criação da cultura organizacional do conhecimento e do comprometimento dos recursos humanos envolvidos [3], [8], [16].

Sistemas de gestão do conhecimento podem ser orientados a processo ou a produto, ou ainda, podem ser a combinação das duas abordagens. Os sistemas orientados a processo oferecem a disseminação do conhecimento, o apoio à discussão e a outros processos de criação do conhecimento, no decorrer da execução dos processos organizacionais. Os sistemas orientados a produto proporcionam a organização do conhecimento em repositórios, de forma a facilitar a sua reutilização em tarefas do dia-a-dia [18], [7].

$\mathrm{O}$ armazenamento e o gerenciamento da informação adquirida por uma empresa ao desempenhar as atividades dos seus processos de negócio devem ser representados na memória organizacional de um sistema de gestão do conhecimento. O conhecimento armazenado, se reutilizado para melhorar o conhecimento sobre o desempenho de uma empresa, pode proporcionar o aprendizado organizacional [4]. Esse aprendizado pode ser apoiado pelo armazenamento e uso de lições aprendidas, visando evitar a repetição dos erros realizados em experiências passadas [19].

O aprendizado organizacional através de um repositório de conhecimento, ocorre quando o profissional realiza as tarefas cotidianas, aprende individualmente, coleta as lições aprendidas e as armazena em um repositório de conhecimento. As lições armazenadas são 
estruturadas de forma a serem facilmente recuperadas e distribuídas para outros interessados, que as aplicarão, promovendo a melhoria dos processos de trabalho. Tendo em vista o processo de aprendizado organizacional através de um repositório de conhecimento, a plataforma $W e b$ oferece algumas vantagens para a implementação de sistemas de gestão do conhecimento [7], [18].

A internet facilita a difusão do conhecimento, ao possibilitar a distribuição uniforme da informação, independentemente da sua forma de armazenamento. Também permite múltiplos servidores de conhecimento, como servidores de: documentos, ontologias, bases de conhecimento, bancos de dados e bibliotecas digitais. Outra característica da Web que oferece vantagens para a gestão do conhecimento é a sua capacidade de horizontalizar o conhecimento na organização. Existem alguns problemas na difusão do conhecimento similares aos problemas enfrentados pelo uso dessa plataforma. Destacam-se as dificuldades em: organizar e indexar a memória organizacional; trazer elementos relevantes que respondam às questões dos usuários; e adaptar a resposta para o usuário aos processos organizacionais. Essas dificuldades podem ser solucionadas por agentes inteligentes ou máquinas de busca na $\mathrm{Web}$ [7].

A literatura de gestão do conhecimento descreve várias abordagens que apóiam a melhoria contínua de processos. Holz et al (2001) propõem uma extensão do ambiente MILOS, que permite modelar e interpretar as necessidades de conhecimento de uma organização durante o planejamento e execução de um projeto de software e, através de um feedback, proporciona melhoria de processos. Lindvall et al (2001) propõem um sistema de gestão de experiências (EMS) e uma metodologia para essa implementação. O EMS é formado por:

- Conteúdo: Dados, informações, conhecimento ou experiência;

- Estrutura: Definição sobre como o conteúdo é organizado e, mais tarde, referenciado como taxonomia ou esquema de classificação;

- Procedimentos: Instruções sobre como gerenciar a base de experiências, incluindo como usar, empacotar, excluir, integrar e atualizar a experiência;

- Ferramentas: Apóiam a gerência do conteúdo e da estrutura e a realização dos procedimentos, assim como, a captura, o armazenamento, a integração, a análise, a sintetização e a recuperação da experiência.

A metodologia sugere os seguintes passos para a implementação de um EMS:

- Caracterizar a organização e definir os seus processos e o conhecimento existente;

- Definir papéis dos usuários (consumidor, mantenedor e provedor) e casos de uso baseados em processos de negócio e nos papéis dos usuários;

- Definir modelo de dados: usado para classificar a experiência a ser incluída no sistema objetivando facilitar a recuperação da experiência;

- Implementar a arquitetura;

- Implantar o sistema e melhorá-lo a partir de feedback. Tipos de feedback:

- Avaliações formais, incluindo entrevistas e teste com os usuários;

- Feedback direto dos usuários;

- Analisar os dados de uso das ferramentas;

- A partir do feedback, atualizar o conteúdo com novas experiências.

Tendo em vista os benefícios proporcionados a uma organização pela gestão do conhecimento, a COPPE/UFRJ iniciou em 2001 o desenvolvimento do meta-ambiente de gestão do conhecimento CORE-KM, que será descrito a seguir. 


\section{O Meta-Ambiente de Gestão do Conhecimento CORE-KM}

O grupo de Engenharia de Software da COPPE/UFRJ está desenvolvendo um metaambiente de gestão do conhecimento, CORE-KM, que pode ser configurado para diferentes organizações de acordo com suas necessidades de conhecimento e com seus processos de negócio específicos. Este meta-ambiente oferece a combinação das duas abordagens de sistemas de gestão do conhecimento, ou seja, é orientado a processo e a produto. CORE-KM apóia a gestão do conhecimento integrado aos processos organizacionais e, também, proporciona a organização do conhecimento em repositórios, facilitando a sua reutilização em atividades do negócio.

CORE-KM é um meta-ambiente baseado na Internet que, após a identificação do perfil de uma organização e de suas necessidades de conhecimento, apóia a configuração de um ambiente de gestão do conhecimento específico para esta organização.

Os ambientes configurados podem ser compostos por diversas ferramentas que apóiem algumas ou todas as seguintes atividades:

- aquisição do conhecimento;

- uso do conhecimento;

- construção do conhecimento;

- disseminação do conhecimento;

- valoração do conhecimento, e,

- manutenção do conhecimento.

Identificadas as necessidades de conhecimento explicito ${ }^{1}$ existente na organização ou fora da mesma, o ambiente configurado apóia a aquisição desse conhecimento, que corresponde à sua coleta estruturada através de ferramenta específica, sua validação e seu armazenamento na memória organizacional.

A utilização do conhecimento consiste no uso do conhecimento para realização das atividades dos processos de negócio da organização. A utilização do conhecimento, proporcionando novos insights sobre como realizar uma determinada atividade e atividades específicas de pesquisa, leva à criação de novos conhecimentos, o que pode constituir atividades específicas voltadas para a construção do conhecimento. A disseminação do conhecimento corresponde à distribuição deste para os integrantes da organização. Essa distribuição deve ser orientada e supervisionada, o que implica na definição sobre quem deve receber qual tipo de conhecimento e em que momento. A valoração do conhecimento é importante para se medir o real valor do conhecimento disponibilizado pelo ambiente para a organização. Para valoração devem ser utilizadas métricas específicas para a avaliação do conhecimento. E, finalmente, a atividade de manutenção do conhecimento, que corresponde à revisão e evolução do conhecimento disponível na memória organizacional, de forma que este seja sempre de valor para a organização.

A figura1 mostra as seis atividades e a infra-estrutura com a memória organizacional do meta-ambiente.

\footnotetext{
${ }^{1}$ Conhecimento explícito corresponde ao que pode ser documentado, definido para um contexto específico e facilmente armazenado e transferido [15].
} 


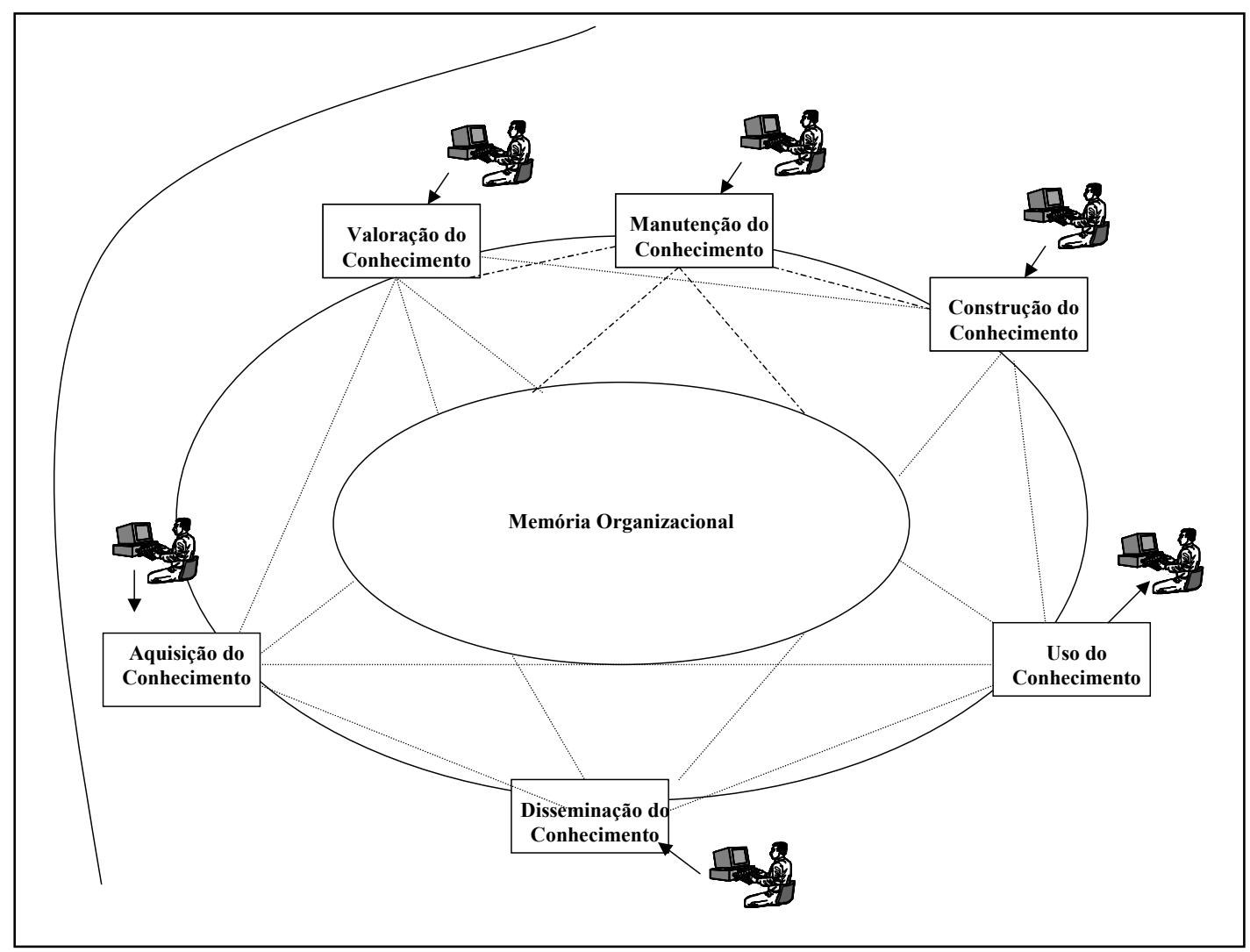

Figura 1: CORE-KM

A infra-estrutura CORE-KM permite que, de acordo com as necessidades particulares de uma empresa, as atividades possam ser exploradas em um ambiente próprio configurado e apoiadas por: repositórios de conhecimento, ontologias, bibliotecas digitais e ferramentas específicas para os processos organizacionais. CORE-KM está sendo, atualmente, configurado para três organizações: a Fundação Bahiana de Cardiologia, o Instituto de Pesquisas da Marinha e a Tecteam Informática. Neste artigo, será descrito o trabalho que está sendo realizado na Tecteam e detalhado o apoio ao processo de fornecimento.

\section{O Ambiente Configurado TecKnowledge}

TecKnowledge é um ambiente de gestão do conhecimento configurado a partir do meta-ambiente CORE-KM para a Tecteam, uma empresa fornecedora de serviços de software.

A definição do ambiente TecKnowledge foi realizada a partir da identificação do conhecimento sobre a organização. Desse conhecimento fazem parte:

- O perfil da empresa, que contém as suas características particulares, áreas de atuação e os processos de negócio, e,

- Os objetivos decisivos, estratégicos, táticos e operacionais de gestão do conhecimento [18].

Ao definir esse trabalho, procurou-se adotar um perfil genérico de uma organização fornecedora de software. Para tanto, o perfil da empresa foi traçado a partir do Processo de Desenvolvimento de Software da ISO/IEC 12207 e da norma NBR ISO 10006 (2000). Essa 
última define diretrizes para a qualidade no gerenciamento de projetos de software e recomenda que sejam utilizados, em todas as atividades do processo, a experiência e os dados históricos provenientes de projetos anteriores. As diretrizes e recomendações propostas na norma NBR ISO 10006 (2000) forneceram os objetivos de gestão do conhecimento para a organização nessa abordagem.

A identificação do perfil da empresa e dos objetivos permitiu que o ambiente TecKnowledge fosse definido para apoiar cinco das seis atividades previstas no metaambiente CORE-KM:

- aquisição do conhecimento;

- uso do conhecimento;

- disseminação do conhecimento;

- valoração do conhecimento, e,

- manutenção do conhecimento.

A atividade de construção do conhecimento não será contemplada no ambiente TecKnowledge, pois a empresa não realiza atividades de pesquisa, mas somente, de desenvolvimento.

Definidas as atividades de gestão do conhecimento a serem apoiadas pelo ambiente, criou-se a memória organizacional do TecKnowledge. A memória organizacional é composta pelo conhecimento associado às atividades do processo intensas em conhecimento. A formalização desse conhecimento no TecKnowledge ocorre através de repositórios de: dados, documentos, melhores práticas e lições aprendidas.

O repositório de dados pode armazenar informações sobre: projetos anteriormente desenvolvidos pela organização, clientes da empresa, entre outras informações sobre os processos de negócio.

O repositório de documentos pode armazenar: propostas, planos de projetos, planos de qualidade e outros documentos sugeridos no Processo de Desenvolvimento de Software da ISO/IEC 12207.

O repositório de melhores práticas pode armazenar conhecimento capturado dos melhores processos de trabalho da organização. Correspondem às atividades de benchmarking que sugerem uma maneira efetiva e eficiente de realizar determinada tarefa.

O repositório de lições aprendidas pode armazenar experiências adquiridas em projetos anteriores. As lições podem ser de três tipos: informacional, bem sucedida ou problema. A lição informacional descreve como agir em algumas situações de emergência. A bem sucedida se refere a ações de sucesso diante de uma crise. E a lição problema ocorre quando uma ação não é bem sucedida na solução de uma questão [17].

A partir da definição da memória organizacional, foram estabelecidos grupos de usuários do conhecimento. Esses grupos foram divididos de acordo com as necessidades de conhecimento de seus componentes. Cada grupo tem acesso ao conhecimento interessante ao seu desempenho nos processos de negócio. Os componentes de cada grupo ainda podem ter permissões diferenciadas de leitura e/ou edição de conhecimento. Também foram definidos papéis para gerentes do conhecimento que são os responsáveis por todo o conhecimento armazenado na memória organizacional. Os gerentes do conhecimento devem estabelecer objetivos de gestão do conhecimento, identificar o conhecimento e realizar as atividades de valoração e manutenção do conhecimento. Essas últimas atividades são oferecidas pelo ambiente TecKnowledge.

\section{Apoio ao Processo de Fornecimento}


Nesta seção, descreve-se o trabalho realizado para a construção de uma ferramenta de apoio ao processo de fornecimento, integrada ao ambiente de gestão do conhecimento TecKnowledge. Esse processo corresponde a um dos processos fundamentais de ciclo de vida definidos na ISO/IEC 12207.

O Processo de Fornecimento da ISO/IEC 12207 define as atividades do fornecedor de software, ou seja, da organização que fornece o produto de software ao adquirente. $\mathrm{O}$ processo pode ser iniciado tanto por uma decisão de preparar uma proposta para responder a um pedido de proposta de um adquirente, quanto pela assinatura e celebração de um contrato com o adquirente para fornecer o sistema, produto ou serviço de software. O processo continua com a determinação dos procedimentos e recursos necessários para gerenciar e garantir o projeto, incluindo o desenvolvimento e a execução dos planos de projeto até a entrega do sistema, produto ou serviço de software para o adquirente [20].

Segundo Aaron (1999), a forma de articular uma proposta tem um grande efeito sobre o que pode vir a acontecer com ela. Por esse motivo, o foco inicial de nosso esforço concentrou-se na tomada de decisão sobre a preparação de uma proposta em resposta a um pedido de um adquirente e na elaboração, propriamente dita, dessa proposta. Nesse processo, as atividades Iniciação e Preparação da Resposta do Processo de Fornecimento de Software, definidas na ISO/IEC 12207 (1998), são apoiadas pela ferramenta TecProposal.

A figura2 descreve o Processo de Fornecimento da ISO/IEC12207, destacando-se as atividades apoiadas por TecProposal. O processo foi modelado segundo a notação proposta em [5]. A notação está sendo utilizada pelo grupo da COPPE e sendo evoluída conforme apresentado no anexo 1.

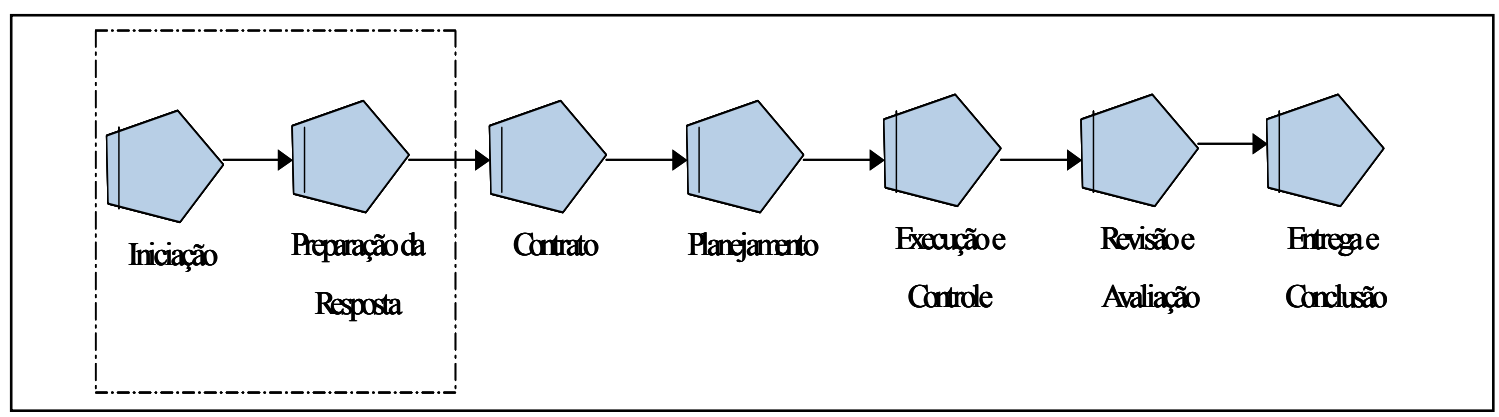

Figura 2: Processo de Fornecimento (ISO/IEC12207, 1998)

A elaboração de propostas técnicas é um processo intenso em conhecimento, normalmente, realizado pelo gerente de projeto de software. Para a sua execução, o gerente deve: conhecer quais informações devem estar contidas na proposta, saber se comunicar através da linguagem escrita e entender do negócio das empresas fornecedora e adquirente de software. Este conhecimento aliado a habilidades específicas desejáveis para o gerente pode garantir o entendimento por parte do adquirente sobre o que está sendo proposto, facilitando a avaliação da proposta. Além disso, a elaboração de propostas requer do gerente o conhecimento sobre o mercado, em termos de preço, tempo e qualidade de produto, e sobre características e particularidades do projeto a ser desenvolvido. Conhecendo esse cenário, o estabelecimento de preços e prazos pode se tornar mais simples e preciso. Outra característica importante para um gerente de projeto de software na elaboração de propostas é a capacidade de realizar a análise de viabilidade do projeto. A partir do conhecimento adquirido em outros projetos, o gerente deve avaliar se o projeto proposto é viável ou não. Esse conhecimento, então, deve apoiar a decisão sobre a elaboração ou aborto da proposta. Sendo a decisão da 
organização pelo projeto afirmativa, o gerente desenvolve o documento com a proposta, finalizando o processo de elaboração de propostas.

A cada proposta elaborada procura-se informação em: propostas, formatos, templates, listas de verificação, diagramas e diretrizes de outros projetos. Além disso, são utilizadas experiências adquiridas pelos gerentes ao desenvolverem outras propostas e ao gerenciarem outros projetos. Essas experiências, na maioria das vezes, não se encontram documentadas [13].

TecProposal apóia, a realização das atividades Iniciação e Preparação da Resposta do Processo de Fornecimento, orientando o gerente na realização das sub-atividades e tarefas envolvidas e disponibilizando o conhecimento necessário armazenado na memória organizacional.

A Iniciação se dá com o fornecedor conduzindo uma revisão dos requisitos que constam do pedido de proposta, levando em consideração políticas e outros regulamentos da organização [20]. O objetivo dessa atividade é o entendimento do problema levantado pelo adquirente na solicitação de proposta, a definição do projeto a ser proposto ao cliente e a tomada de decisão sobre a elaboração ou aborto da proposta.

A figura3 descreve a atividade Iniciação, apoiada pela ferramenta TecProposal e modelada segundo a notação do anexo 1 .

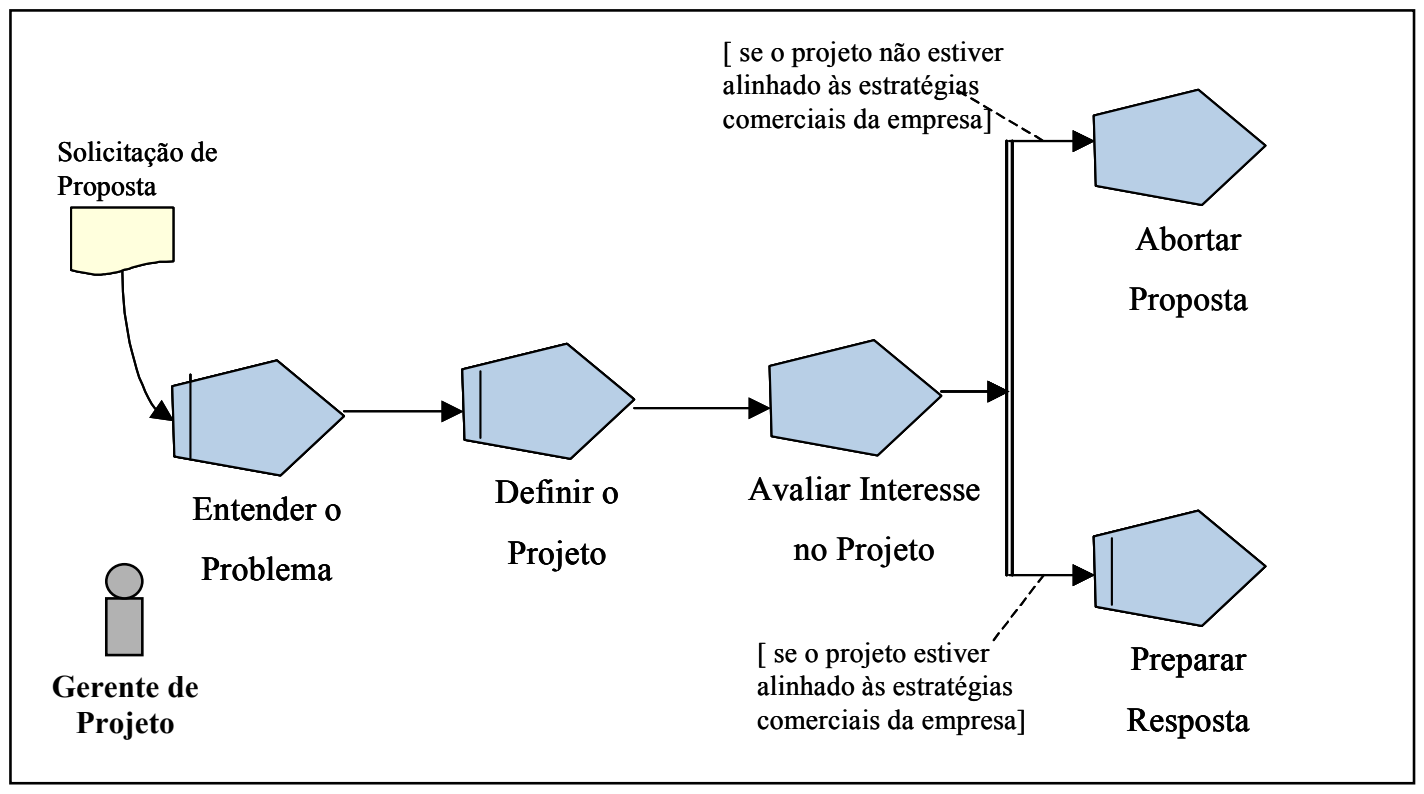

Figura 3: Atividade - Iniciação

A Preparação da Resposta corresponde à definição e à preparação, por parte do fornecedor, de uma Proposta de Projeto em resposta ao pedido de proposta [20]. O objetivo dessa atividade é definir uma solução viável para o problema proposto pelo cliente, estimando esforço, tempo e custos de desenvolvimento. O produto final dessa atividade é a proposta redigida.

A figura4 descreve a atividade Preparação da Resposta, apoiada pela ferramenta TecProposal e modelada segundo a notação do anexo 1. 


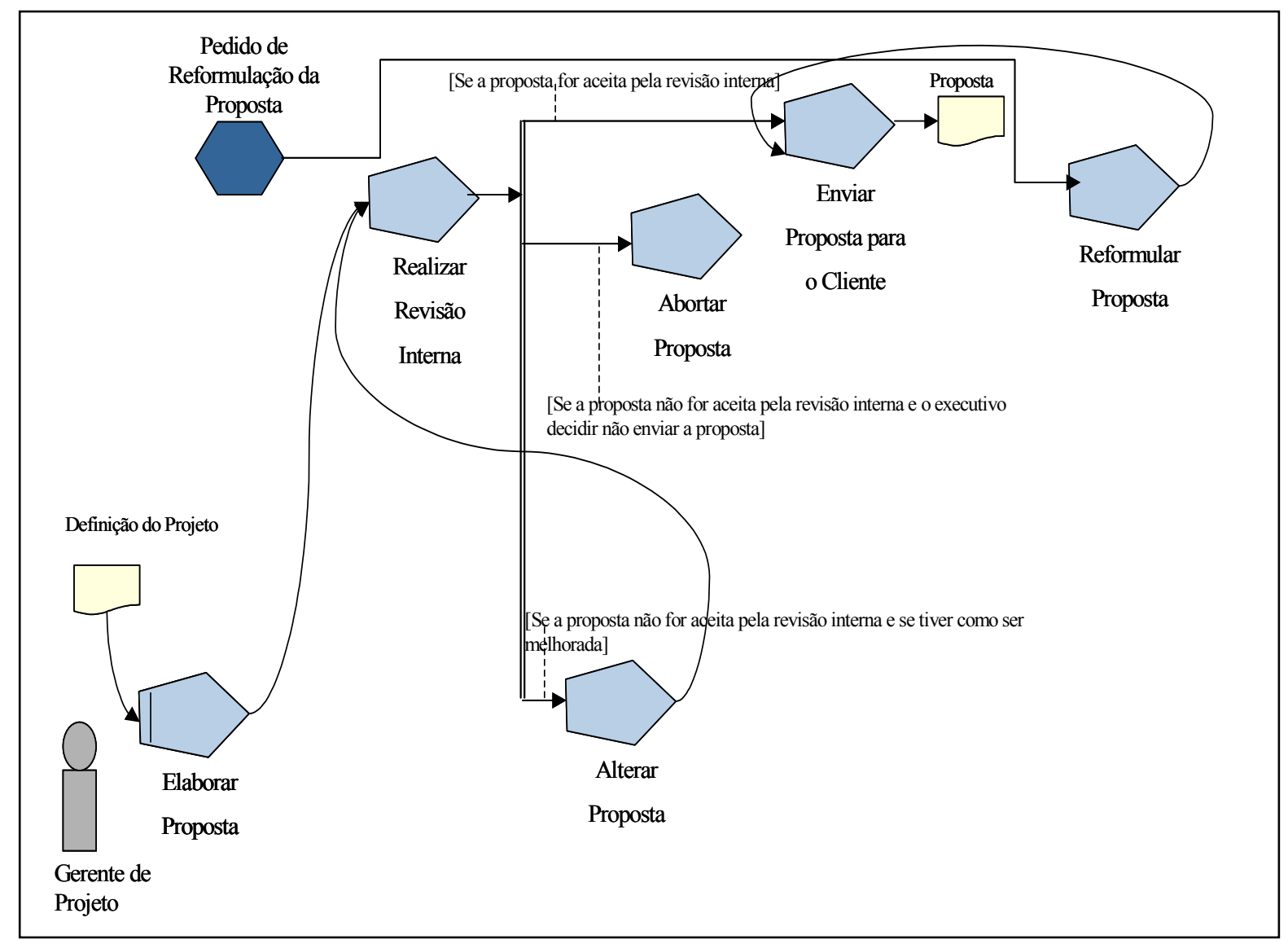

Figura 4: Atividade - Preparação da Resposta

A gestão do conhecimento pode beneficiar gerentes de projetos na rapidez do desenvolvimento de propostas, na tomada de decisão e na melhoria da qualidade do processo de elaboração de propostas. Para tanto, o conhecimento adquirido nas experiências dos gerentes, normalmente, está na forma tácita e deve ser transformado em explícito. A representação explícita do conhecimento facilita o seu entendimento e, então, apóia a tomada de decisão de acordo com os objetivos organizacionais [6], [13].

TecProposal é, assim, uma ferramenta de apoio à utilização do conhecimento no processo de elaboração de propostas para projetos de software, desenvolvida com base no Processo de Fornecimento de Software definido na ISO/IEC 12207 (1998) e na NBR ISO 10006 (2000). TecProposal tem como objetivo o apoio ao gerente de projetos na tomada de decisão sobre pedidos de propostas de projetos e na elaboração de propostas técnicas em resposta ao pedido de proposta de um cliente.

A ferramenta oferece modelos de propostas para padronizar esses documentos e proporcionar a sua elaboração em menor tempo. TecProposal também orienta a elaboração de propostas através de FAQs e de "receitas" de como fazer.

Os repositórios de conhecimento na memória organizacional provêem conhecimento específico à execução do processo de elaboração de proposta, dados históricos de projetos e de propostas anteriores, assim como informações específicas sobre clientes e organizações e documentos de projetos. O conteúdo da memória organizacional consiste em:

- Propostas customizadas: Correspondem a modelos de propostas sugeridos para elaboração do documento. Os modelos podem ser customizados para atender os padrões da organização; 
- Informações sobre os recursos humanos: Consiste em informação completa sobre os recursos humanos da organização, ou seja, são os currículos dos funcionários que trabalham ou já trabalharam em projetos da organização. Oferece um resumo por categoria (analista, programador, gerente de projeto) e por maturidade $(0$ - sem experiência, 1 - treinamento acadêmico, 2 - prática em projeto, 3 - experiente, 4 capaz de orientar outros) para uso na proposta.

- Experiência em projetos: Corresponde ao armazenamento de experiência, em projetos anteriores, relevante às necessidades da proposta. Cataloga todos os elementos importantes em um projeto, incluindo domínio da aplicação, tecnologia utilizada, porte do projeto, o objetivo, a abrangência e o pré-escopo do projeto, esforço, tempo e custos de desenvolvimento, informações sobre o cliente., etc.

- Experiência em propostas: Consiste em números de propostas elaboradas e de propostas que se converteram em contratos, motivos para rejeição de propostas (tanto por clientes como internamente), tempo de resposta a uma proposta e freqüência de solicitação de proposta por um cliente.

- Parceiras: São informações sobre empresas que possuem relacionamento de parceria com a utilizadora do TecProposal. Lista as características e especialidades da parceira, o status da parceria e o histórico de projetos juntos.

- FAQs: Cataloga as perguntas normalmente feitas durante a elaboração de uma proposta.

- "Receitas" de como fazer: Descreve os passos para elaboração de uma proposta.

Depois da proposta elaborada, essa é enviada ao cliente, que avalia o seu conteúdo. A proposta é avaliada e, em caso de sucesso, o cliente propõe um contrato com a empresa fornecedora. Caso contrário, a organização que elaborou a proposta deveria procurar descobrir e documentar qual foi a proposta vencedora da concorrência e quais os critérios da escolha. Esse conhecimento visa a melhoria do processo de elaboração de propostas da organização.

TecProposal formaliza o conhecimento sobre propostas adquirido por gerentes de projetos em suas experiências pessoais e proporciona o aprendizado organizacional. Esse aprendizado é realizado, à medida em que, a ferramenta apóia a busca e a recuperação de conhecimento armazenado nos seus repositórios e oferece a melhoria do processo de elaboração de propostas de projetos de software. É importante ressaltar que uma ferramenta como a TecProposal não garante a aceitação de uma proposta por um cliente, mas visa aumentar as chances de acordo entre as partes envolvidas.

\section{Conclusões}

A tomada de decisão sobre pedidos de propostas de projetos e a elaboração de propostas técnicas em resposta ao pedido de proposta de um cliente são atividades intensas em conhecimento. A decisão sobre responder um pedido de proposta considera se o projeto alinha-se aos objetivos e estratégias comerciais da empresa. No caso do projeto ser avaliado como interessante para a empresa, realiza-se a elaboração da proposta de projeto. Caso contrário, aborta-se a proposta, documentando-se os motivos da decisão. Desse modo, a tomada de decisão necessita de conhecimento prévio, de forma intensiva.

A elaboração da proposta, ou Preparação da Resposta do Processo de Fornecimento, também é uma atividade intensa em conhecimento sobre projetos e propostas anteriores. Além de exigir do gerente de projeto conhecimento sobre o cliente. 
Para apoiar essas atividades críticas ao desenvolvimento de software, foi proposta a TecProposal, uma ferramenta de gestão do conhecimento relativo à elaboração de propostas de projetos de software.

$\mathrm{Na}$ literatura sobre propostas de projetos de software [11] e sobre o processo de fornecimento [9], [14], observou-se que o uso, em todas as atividades do processo, da experiência e dos dados históricos provenientes de projetos anteriores pode melhorar a qualidade do processo.

TecProposal está em fase final de desenvolvimento e validação na Tecteam Informática. É uma ferramenta integrada ao ambiente TecKnowledge, configurado a partir do meta-ambiente CORE-KM. O TecKnowledge foi desenvolvido para a intranet da Tecteam, seguindo a configuração para o perfil da empresa, sugerida pelo CORE-KM. A implementação foi em ASP.net e SQL Server, o que facilita a distribuição uniforme da informação e permite múltiplos servidores de conhecimento. A configuração do ambiente TecKnowledge mostrou ser esta uma solução adequada para construção de ambientes de gestão do conhecimento para empresas desenvolvedoras de software.

\section{Referências}

[1] Aaron, M.C., "The Right Frame: Managing Meaning and Making Proposals", Harvard Management Communication Letter Article, setembro/1999.

[2] A.Abecker, A.Bernardi, M.Sintek, "Developing a Knowledge Management Technology - an Encompassing View on KnowMore, Know-Net, and Enrich", In: Proceedings of the IEEE WET-ICE'99 Workshop on Knowledge Media Networking, Stanford, Junho/1999.

[3] M.Alavi, D.Leidner, "Knowledge Management Systems: Emerging Views and Practices from the Field", IEEE 1999, Proceedings of the $32^{\text {nd }}$ Hawaii International Conference on System Sciences - 1999.

[4] M.Berger, et al, "A Learning Component for Workflow Management Systems", $31^{\text {st }}$ Hawaii International Conference on System Science, 1998

[5] C.S.Bonfim, "Uma Ferramenta de Modelagem de Processos para Ambientes de Desenvolvimento de Software Orientados à Organização", Monografia de Final de Curso, Curso de Ciência da Computação da Universidade Federal da Bahia, Setembro de 2001.

[6] C.W.Choo, "Knowing Organization: How Organizations Use Information to Construct Meaning, Create Knowledge and Make Decisions", New York: Oxford University Press, 1998.

[7] Dieng, R. , "Knowledge Management and the Internet", IEEE Intelligent Systems, Vol. 15, No. 3, May/June2000, pp $14-17$

[8] G.Fischer, J.Ostwald, "Knowledge Management: Problems, Promises, Realities, and Challenges", IEEE Intelligent Systems, January/February 2001, pp.60-72

[9] H.Holz et al, "Task-Specific Knowledge Management in a Process-Centred SEE", K.D.Althoff, R.L. Feldman, and W.Muller (Eds.): LSO2001, LNCS2176, pp-163/177, 2001.

[10] "ISO/IEC 12207: Tecnologia de Informação - Processos de Ciclo de Vida de Software", 1998.

[11] P.Jalote, "CMM in Practice - Processes for Executing Software Projects at Infosys", Addison Wesley Longman, Inc., 2000. 
[12] M.Lindvall et al, "Lessons Learned about Structuring and Describing Experience for Three Experiences Bases", K.-D.Althoff, R.L. Feldman, and W.Muller (Eds.): LSO2001, LNCS2176, pp-106/119, 2001.

[13] R.Murch, "Project Management - Best Practices from IT Professionals", $1^{\text {st }}$ Edition, Prentice Hall, ISBN: 0-13-021914-2

[14] “NBR ISO 10006: Gestão da Qualidade - Diretrizes para a Qualidade no Gerenciamento de Projetos", 2000.

[15] I.Nonaka, H.Takeuchi, "Criação de conhecimento na empresa: como as empresas japonesas geram a dinâmica da inovação", Rio de Janeiro: Editora Campus, 1997.

[16] D.E.O'Leary, “Knowledge Management Systems: Converting and Connecting”, Intelligent Systems IEEE, v.13, n.3 (May/Jun1998), pp.30-33

[17] D.E.O'Leary, “Enterprise Knowledge Management”, IEEE Computer, March 1998, pp.54-61

[18] B.Snoek, "Knowledge Management and Organizational Learning - Systematic Development of an Experience Base on Approaches and Technologies", Diploma Thesis, Fraunhofer Einrichtung Experimentelles Software Engineering, setembro/1999.

[19] J. Statz, "Leverage your Lessons", IEEE Software, March/April 1999, pp.30-32

[20] A.R.Rocha, J.C.Maldonado, K.C.Weber, "Qualidade de Software - Teoria e Prática", São Paulo: Prenctice Hall, 2001, pp.39-43.

[21] Winch, G., “Knowledge Management". Manufacturing Engineer, pages 178-180. August 1999. 
Anexo 1: Notação da Modelagem de Processos

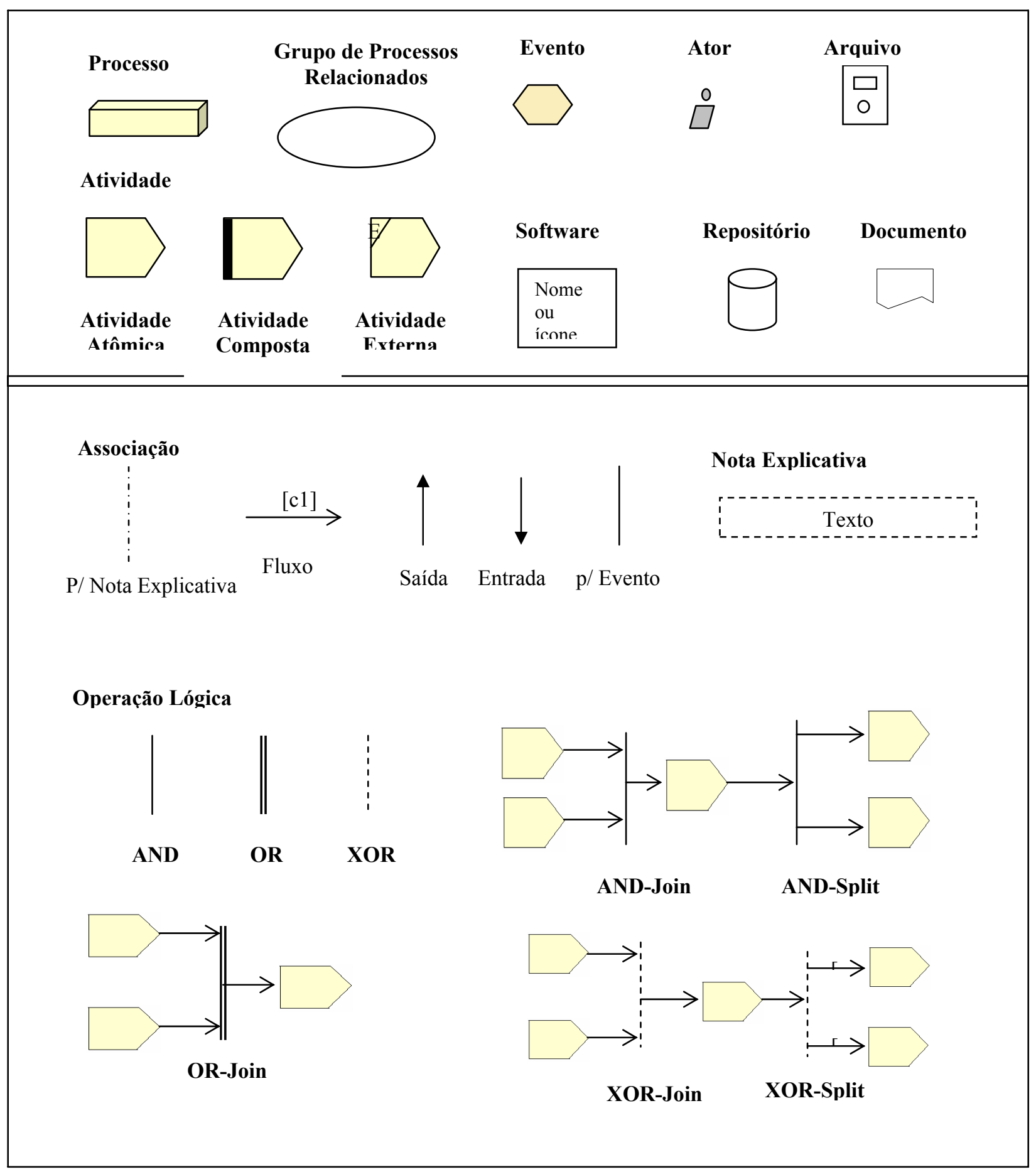

